\title{
FULL SPARK FRAMES IN THE ORBIT OF A REPRESENTATION
}

\author{
ROMANOS DIOGENES MALIKIOSIS, VIGNON OUSSA
}

\begin{abstract}
We present a new infinite family of full spark equal norm tight frames in finite dimensions arising from a unitary group representation, where the underlying group is the semi-direct product of a cyclic group by a group of automorphisms. The only previously known algebraically constructed infinite families were the harmonic, Gabor and Dihedral frames. Our construction hinges on a theorem that requires no group structure. Additionally, we illustrate our results by providing explicit constructions of full spark frames.
\end{abstract}

\section{INTRODUCTION}

The notion of a full spark frame is of fundamental importance in the theory of signal processing and relevant in areas such as compressed sensing and quantum information theory. Although the notion of a frame was first defined and used in infinite-dimensional Hilbert spaces, its finite counterpart took over in significance, especially in applications, and this is precisely the setting of the current article. Most notably, we mention the solution to the Kadison-Singer problem [14] and the SIC-POVM existence problem [20]; the first problem can be formulated in the finite frame setting and has implications to diverse areas of mathematics, such as operator theory and Banach space theory [4], while the second problem seems to indicate the existence of a profound connection between quantum information theory and algebraic number theory, especially Hilbert's 12th problem [2]. For a comprehensive introduction to finite frames, we refer the reader to the books [5, 19].

We will briefly describe the motivation behind the search for full spark frames, that arises from applications. In a finite-dimensional complex vector space, say $\mathbb{C}^{N}$, a frame is just a finite spanning set. We consider the following setting: suppose that we want to transmit a vector $v \in \mathbb{C}^{N}$, through a channel with erasures, i.e., some information packets are lost to the recipient. We try to find an optimal way, to ensure that the recipient will have all the necessary information to recover the initial vector $v$.

Transmitting the coordinates of $v$ is not a good strategy, because we cannot recover $v$ even if just one coordinate is lost. Similarly, transmitting each coordinate of $v$ a number of times (i.e., we send every coordinate to the recipient 100 times) is also not a good strategy. Recoverability of $v$ significantly increases when we send inner products $\left\langle\varphi_{j}, v\right\rangle$, where $\Phi=\left\{\varphi_{1}, \ldots, \varphi_{M}\right\} \subseteq \mathbb{C}^{N}$ is a spanning set of $\mathbb{C}^{N}$. We want to choose $\Phi$ in such a way, so that every $N$ vectors of $\Phi$ form a basis of $\mathbb{C}^{N}$. When this happens, we can recover $v$ from any $N$ inner products. For this reason, a set $\Phi$ with this property is also named maximally robust to erasures. This means that no matter which $M-N$ inner products are lost, we can still recover $v$. 
This is precisely the definition of a full spark frame. Picking $M$ vectors at random (say, independently and uniformly from a Gaussian distribution) will yield a full spark frame with probability 1 , as long as $M>N$. However, verifying whether a given set of vectors is full spark is an NP-hard problem [1]. Moreover, a random selection of vectors provides absolutely no control over other characteristics of a frame, such as tightness, coherence, condition number, uniformity of norm, etc. For this purpose, it is important to build such frames algebraically (i.e., in a deterministic way), with prescribed properties.

On the one hand, observe that any frame is canonically associated with a tight frame obtained by applying the inverse of the square-root of the frame operator to it. Additionally, full spark frames exist in abundance [1, 3], and as such, full spark tight frames can be easily constructed. On the other hand, full spark equal norm tight frames are much harder to come by, and representation theory provides powerful tools that can be exploited to construct such frames in a systematic fashion. From a representation theoretic perspective, the following question arises naturally. Is it possible to characterize finite-dimensional and unitary representations of finite groups for which, there exists a vector whose orbit contains a full spark equal norm and tight frame? To the best of our knowledge, this is a difficult question which has not been systematically studied in the literature. However, several concrete representations have been thoroughly investigated. For instance [11, Lawrence, Pfander, and Walnut considered the action of a unitary and irreducible representation of a finite Heisenberg group acting on an $n$-dimensional vector space $n \geq 2$, when the underlying group is cyclic. Any frame contained in the orbit of a fixed vector is called a finite-dimensional Gabor frame. Such a representation is a finite-dimensional analog of the Schrödinger representations, which are known to be the group-theoretic foundation of Gabor Theory [6, 10]. Regarding the problem of existence of finite-dimensional Gabor tight frames, which are full spark, Lawrence, Pfander, and Walnut gave some partial solution in [11. Precisely, they proved that in the case where $n$ is prime, there exists an orbit containing a full spark frame consisting of $n^{2}$ vectors. In fact, the set of all vectors generating such a frame is Zariski open and dense in $\mathbb{C}^{n}$. This problem was eventually completely settled by the first author of the current paper (see [12]) who not only established the existence of tight Gabor frames for all natural numbers $n \geq 2$ but also gave a deterministic construction. When the underlying group of the Heisenberg group is abelian, non-cyclic, the first author showed that the Gabor frames under question can never be full spark [13].

In another direction, the second author of this paper and Sheehan considered a class of quasiregular representations of the Dihedral group and investigated the existence and explicit construction of full spark frames [16, 17] associated with such representations. Surprisingly, their investigation revealed that the existence of full spark frames is a function of the parity of the dimension of the representations. Precisely, it is shown in [17 that there exists an orbit, which is an equal norm full spark frame if and only if the dimension of the quasiregular representation is odd. Additionally, an algorithmic construction of such frames can be found in [17].

The present paper is organized as follows: in Section 3, we define representations of finite groups that are full spark or spark deficient, and give some criteria for spark deficient groups. Next, in Section 4, we focus on representations of semi-direct products of cyclic groups by a subgroup of automorphisms, and provide some new infinite families of full 
spark frames, via the existence of full spark groups. The main technique generalizes the method used in a previous article of the first-named author [12]. Finally, in Section 5 we extend these results by noting that an underlying group structure is not always necessary to produce full spark tight frames, and provide some examples.

\section{Notation, terminology, And General RESUlts}

Let $\mathcal{H}$ be a finite-dimensional Hilbert space equipped with an inner product which we shall denote by $\langle\cdot, \cdot\rangle$. Recall that a finite sequence of vectors $\left(v_{k}\right)_{k \in I}$ is called a frame for $\mathcal{H}$ if there exist positive real numbers $A \leq B$ such that

$$
A\|w\|^{2} \leq \sum_{k \in I}\left|\left\langle w, v_{k}\right\rangle\right|^{2} \leq B\|w\|^{2}
$$

for all vectors $w \in \mathcal{H}$. The constants $A, B$ are called the lower frame bound and upper frame bound of $\left(v_{k}\right)_{k \in I}$ respectively. In cases where the two bounds coincide, we call $\left(v_{k}\right)_{k \in I}$ a tight frame. Thanks to Cauchy-Schwarz inequality, it is clear that any finite collection of vectors has an upper frame bound. In fact, since we are only considering finite-dimensional vector spaces, it is not difficult to verify that a collection of vectors $\left(v_{k}\right)_{k \in I}$ has a lower frame bound if and only if it spans $\mathcal{H}$.

Any frame $\left(v_{k}\right)_{k \in I}$ for $\mathcal{H}$ gives rise to three important operators: the analysis, synthesis and frame operators. The analysis operator is given by the map

$$
C: w \mapsto\left(\left\langle w, v_{k}\right\rangle\right)_{k \in I} \in l^{2}(I), w \in \mathcal{H}
$$

Its adjoint is the synthesis operator, $C^{*}:\left(c_{k}\right)_{k \in I} \mapsto \sum_{k \in I} c_{k} v_{k} \in \mathcal{H}$, and the operator

$$
S=C^{*} C: w \mapsto \sum_{k \in I}\left\langle w, v_{k}\right\rangle v_{k}
$$

obtained by composing the analysis and the synthesis operators defines a self-adjoint map on $\mathcal{H}$ known as the frame operator. It is a standard fact in frame theory that $\left(v_{k}\right)_{k \in I}$ is a frame if and only if the frame operator $S$ is invertible [5, 19]. Note also that $S$ is invertible if and only if the analysis operator $C$ is injective. Moreover, if $\left(v_{k}\right)_{k \in I}$ is a frame for $\mathcal{H}$, then every vector $w \in \mathcal{H}$ admits admits an expansion of the type

$$
w=\sum_{k \in I}\left\langle w, S^{-1} v_{k}\right\rangle v_{k}=\sum_{k \in I}\left\langle w, v_{k}\right\rangle S^{-1} v_{k}
$$

Generally, since (2.1) requires inverting the frame operator, the process of reconstructing a vector $w$ from the coefficients $\left\langle w, v_{k}\right\rangle, k \in I$ can be computationally expensive. However, for the special case where $\left(v_{k}\right)_{k \in I}$ is a tight frame, the frame operator is just a multiple of the identity map, and in that case (2.1) simply becomes

$$
w=\frac{1}{A} \sum_{k \in I}\left\langle w, v_{k}\right\rangle v_{k} .
$$

For this reason, tight frames are more appealing than non-tight ones. 
2.1. Unitary representations of finite groups. Let $G$ be a finite group. A unitary representation $\pi$ is a homomorphism which maps $G$ into the group $\mathcal{U}\left(\mathcal{H}_{\pi}\right)$ of unitary operators acting in some Hilbert space $\mathcal{H}_{\pi}$. We say that $\pi$ is an $n$-dimensional representation of $G$ if $\mathcal{H}_{\pi}$ is an $n$-dimensional vector space over the field of complex numbers.

A representation is said to be faithful if its kernel is trivial. Moreover, two unitary representations $\pi$ and $\tau$ of $G$ acting on $\mathcal{H}_{\pi}$ and $\mathcal{H}_{\tau}$ respectively, are unitarily equivalent if there exists a unitary operator $J: \mathcal{H}_{\pi} \rightarrow \mathcal{H}_{\tau}$ such that $J \pi(x) J^{-1}=\tau(x)$ for all $x \in G$.

Let $\mathcal{K}$ be a vector subspace of $\mathcal{H}_{\pi}$. We say that $\mathcal{K}$ is $\pi$-invariant if for every $x \in G$, $\pi(x) \mathcal{K}$ is a subset of $\mathcal{K}$. Associated to any $\pi$-invariant subspace of $\mathcal{H}_{\pi}$, is a unitary representation $\pi_{\mathcal{K}}$ of $G$ obtained by restricting the action of $\pi$ to $\mathcal{K}$ as follows: $\pi_{\mathcal{K}}(x)=\pi(x) \mid \mathcal{K}$ for all $x \in G$. Such a representation is called a subrepresentation of $\pi$. Any representation which admits a nontrivial subrepresentation is called reducible. Otherwise, such a representation is called irreducible. Irreducible representations of finite groups play a central role in their representation theory since they form the basic building blocks of all unitary representations. For this reason, an important aspect of representation theory of finite groups consists of classifying up to unitary equivalence, all irreducible representations of a given finite group.

A representation $\pi$ of a finite group $G$ acting in a finite-dimensional Hilbert space $\mathcal{H}_{\pi}$ is said to be cyclic if there exists at least one vector $v \in \mathcal{H}_{\pi}$ such that $\pi(G) v$ spans $\mathcal{H}_{\pi}$.

The left action of any group on itself, induces an important unitary representation known as the left regular representation $L$ of $G$ which acts on the Hilbert space $l^{2}(G)$ as follows. Given $f \in l^{2}(G)$, and $x, y \in G, L(x) f(y)=f\left(x^{-1} y\right)$. The regular representation of a finite group is known to be the mother of all representations since any irreducible representation of $G$ is unitarily equivalent to a subrepresentation of the left regular representation (see [18, Chapter 3]).

2.2. Group full spark frames. Let $\pi$ be unitary representation of a finite group acting in a finite-dimensional vector space. Then $\pi$ admits a cyclic vector (a vector whose orbit is a spanning set), if and only if $\pi$ is equivalent to a subrepresentation of the regular representation [19]. Thus, the study of representations giving rise to frames can be essentially reduced to the study of subrepresentations of the regular representation.

Definition 2.1. Let $G$ be a finite group and let $\pi: G \rightarrow \mathcal{U}(\mathcal{H})$ be a unitary and cyclic representation acting on some finite-dimensional Hilbert space $\mathcal{H}$. We say that the representation $\pi$ is spark deficient if for every vector $v \in \mathcal{H}$, the sequence $(\pi(g) v)_{g \in G}$ is spark deficient (it is not a full spark frame.) Moreover, we say that $\pi$ is full spark if there is a vector $v \in \mathcal{H}$ such that $(\pi(g) v)_{g \in G}$ is a full spark frame. Such a vector when it exists will be called a $\pi$-full spark vector.

It is worth noting if a representation is full spark then the set of $\pi$-full spark vectors is a Zariski open and dense subset of the Hilbert space on which the representation is acting. 
Definition 2.2. If $G$ is a finite group such that every irreducible unitary representation of $G$ which is not a character is spark deficient, we call $G$ an irreducibly spark deficient group. On the other hand, we shall say that $G$ is irreducibly full spark if every unitary irreducible representation of $G$ is full spark.

Remark 1. Our investigation seems to reveal that non abelian irreducibly full spark groups are quite rare. Moreover, it is worth highlighting that there do exist groups which are neither irreducibly full spark nor irreducibly spark deficient. For instance, let $D_{2 N}$ be the Dihedral group of order $2 N, N \geq 3$. Then it is known that [16] such a group is irreducibly full spark if $N$ is prime, irreducibly spark deficient if $N$ is even, and neither if $N$ is odd and composite. Furthermore, since every abelian group is trivially irreducibly full spark, the classification of irreducibly full spark groups is only interesting for nonabelian groups.

Recall that for a finite group $G$, the convolution $*$ product is defined as follows. Given complex-valued functions $f, g$ defined on $G$,

$$
f * g(x)=\sum_{y \in G} f(y) g\left(y^{-1} x\right) .
$$

Let $\pi$ be a unitary irreducible representation of $G$ acting in some Hilbert space $\mathcal{H}$. Next, for a fixed nonzero vector $\phi \in \mathcal{H}$, let $V_{\phi}: \mathcal{H} \rightarrow l^{2}(G)$ be a linear operator defined as follows: $V_{\phi} \psi(x)=\langle\psi, \pi(x) \phi\rangle$ for $\psi \in \mathcal{H}$. Put

$$
\mathcal{C}_{\pi}=\{c: G \rightarrow \mathbb{C}:|\operatorname{supp}(c)|=\operatorname{dim}(\mathcal{H})\} .
$$

Our first result in this section, gives a complete characterization of $\pi$-full spark frame vectors in terms of the convolution product on $G$. Precisely, it states that $\phi$ is a $\pi$-full spark vector if and only if no element of $\mathcal{C}_{\pi}$ is a left zero divisor of $V_{\phi} \phi$ with respect to the convolution operation on $G$.

Proposition 2. $\phi$ is a $\pi$-full spark vector if and only if for any $c \in \mathcal{C}_{\pi}, c * V_{\phi} \phi$ is a nonzero sequence.

Proof. Suppose that there exists a vector $f$ in the null-space of $V_{\phi}$. That is, $\langle f, \pi(x) \phi\rangle=$ 0 for all $x \in G$. Since $\pi$ is irreducible, $f$ must be the zero vector. Thus, $V_{\phi}$ is an injective linear operator and consequently, $(\pi(x) \phi)_{x \in G}$ is a frame for the Hilbert space $\mathcal{H}$. Moreover, the frame operator $V_{\phi}^{*} V_{\phi}: \mathcal{H} \rightarrow \mathcal{H}$ is defined as follows. Given $\psi \in l^{2}(G)$, we have $V_{\phi}^{*} V_{\phi} \psi=\sum_{x \in G}\langle\psi, \pi(x) \phi\rangle \pi(x) \phi$. Furthermore, for arbitrary $y \in G$, it is clear that $V_{\phi}^{*} V_{\phi} \pi(y) \psi=\pi(y) V_{\phi}^{*} V_{\phi} \psi$. Thus, the linear operator $V_{\phi}^{*} V_{\phi}$ is in the commutant of the algebra generated by $\pi$. Since $\pi$ is irreducible, according to Schur's lemma, the linear operator $V_{\phi}^{*} V_{\phi}$ must be a constant multiple of the identity map and consequently, $(\pi(x) \phi)_{x \in G}$ must be a tight frame for $\mathcal{H}$. This implies that $V_{\phi}$ defines (up to multiplication by a constant) an isometry between $\mathcal{H}$ and its image $V_{\phi}(\mathcal{H})$. Observing that $V_{\phi}(\mathcal{H})$ is a left-invariant vector space and letting $\lambda$ be the restriction of the left regular representation of $G$ to $V_{\phi}(\mathcal{H})$, it follows that $V_{\phi}$ intertwines $\pi$ with $\lambda$. As such, it is clear that $(\pi(x) \phi)_{x \in G}$ is a full spark tight frame for $\mathcal{H}$ if and only if $\left(\lambda(x) V_{\phi} \phi\right)_{x \in G}$ is a full spark tight frame for the Hilbert space $V_{\phi}(\mathcal{H})$. However, the latter statement holds if for arbitrary $c \in \mathcal{C}_{\pi}, 0 \neq \sum_{x \in G} c(x) \lambda(x) V_{\phi} \phi$. The stated result follows then from the observation that for arbitrary $y \in G, \sum_{x \in G} c(x) V_{\phi} \phi\left(x^{-1} y\right)=c * V_{\phi} \phi(y)$. 
Proposition 3. Let $\pi$ be a finite-dimensional representation of a finite group $G$ acting in a Hilbert space $\mathcal{H}$. Then $\pi$ is full spark with a $\pi$-full spark vector $\phi$ if and only if for any nonzero vector $\psi \in \mathcal{H}$, the sequence $(\langle\psi, \pi(x) \phi\rangle)_{x \in G}$ has at most $\operatorname{dim}(\mathcal{H})-1$ components which are equal to zero.

Proof. Let us suppose that $\pi$ is full spark with full spark vector $\phi$. For any subset $X$ of $G$ of cardinality $\operatorname{dim}(\mathcal{H})$, let $V_{\phi, X}: \psi \mapsto(\langle\psi, \pi(x) \phi\rangle)_{x \in X}$ be the analysis operator corresponding to the frame $(\pi(x) \phi)_{x \in X}$. Since for any nonzero vector $\psi \in \mathcal{H}$ the sequence $(\langle\psi, \pi(x) \phi\rangle)_{x \in X}$ is nonzero in $l^{2}(X)$, then $(\langle\psi, \pi(x) \phi\rangle)_{x \in G}$ has at most $\operatorname{dim}(\mathcal{H})-1$ components which are equal to zero. Since the converse of the previous statement follows from similar arguments, we omit its proof.

\section{SPARK DEFICIENT REPRESENTATIONS}

Throughout this section, unless we state otherwise, all representations under consideration are assumed not to be unitary characters.

The following facts are straightforward yet essential observations.

(a) Let $\pi$ be a unitary irreducible representation of $G$. If there exists a finite subset $X$ of $G$ of cardinality at most $\operatorname{dim} \mathcal{H}$ such that the collection of unitary operators $\pi(x), x \in X$ is linearly dependent in the vector space of linear operators acting on $\mathcal{H}$ then $\pi$ is spark deficient.

(b) Let $\pi$ be a unitary representation of a finite group $G$ acting in a finite-dimensional Hilbert space $\mathcal{H}$ such that $|G| \geq \operatorname{dim}(\mathcal{H})>1$. If $\pi$ is not faithful then it must be spark deficient. Indeed, if $\pi$ is assumed not to be faithful, then there exists a non-trivial element $x$ in its kernel, which maps to the identity operator acting on $\mathcal{H}$. Letting $e$ be the identity in $G$, we obtain that $\pi(e)-\pi(x)$ is the zero operator.

3.1. The spark deficiency of the direct sum of two equivalent full spark representations. Since irreducible representations are the building blocks of all representations, the following question is quite natural. Given a full spark irreducible representation, is the representation obtained as the direct sum of copies of the given representation full spark as well? The result below answers this question negatively under some natural assumptions.

Proposition 4. Let $G$ be a finite group and let $\pi$ be a faithful unitary representation of $G$ acting in $\mathcal{H}$ for which there exists an element $x \in G$ such that the order of $\pi(x)$ is at least twice of the dimension of $\mathcal{H}$. Then $\pi \oplus \pi$ must be spark deficient.

Proof. Suppose by contradiction that $\tau=\pi \oplus \pi$ is full spark. Then there exists $\phi \in$ $\mathcal{K}=\mathcal{H} \oplus \mathcal{H}$ such that $(\tau(g) \phi)_{g \in G}$ is a full spark frame for $\mathcal{K}$. Next, let $x \in G$ such that the order of $\pi(x)$ is at least twice of the dimension of $\mathcal{H}$. Since, every eigenvalue of $\tau(x)$ occurs at least twice, there exists an invertible matrix $Q$ such that $D=Q \tau(x) Q^{-1}$ is a diagonal matrix with diagonal entries listed in order as follows (including multiplicity): 
$\lambda, \lambda, \lambda_{1}, \cdots, \lambda_{N-2}$, such that $|\lambda|=\left|\lambda_{1}\right|=\cdots=\left|\lambda_{N-2}\right|=1$. Let $e_{1}, \cdots, e_{N}$ be the canonical orthonormal basis for $\mathcal{K}=\mathbb{C}^{N}$. By assumption, the restriction of $\tau$ to the subgroup generated by $x$ is a cyclic representation of the subgroup and consequently, it is clear that the analysis operator $f \mapsto(\langle f, \tau(y) \phi\rangle)_{y \in\langle x\rangle}$ defines an injection between $\mathcal{K}$ and $l^{2}(\langle x\rangle)$. Next, for any integer $k$, straightforward calculations give

$$
\left\langle f, \tau\left(x^{k}\right) \phi\right\rangle=\left\langle Q^{*}\left(Q^{*}\right)^{-1} f, \tau\left(x^{k}\right) \phi\right\rangle=\left\langle\left(Q^{*}\right)^{-1} f, D^{k} Q \phi\right\rangle .
$$

Moving forward, we shall assume that $\phi$ satisfies the following additional conditions: $Q \phi=\alpha_{1} e_{1}+\alpha_{2} e_{2}+w$ and $w \in \mathbb{C}-\operatorname{span}\left\{e_{3}, \cdots, e_{N}\right\}$. Thus,

$$
\left\langle f, \tau\left(x^{k}\right) \phi\right\rangle=\lambda^{-k} \cdot\left\langle\left(Q^{*}\right)^{-1} f, \alpha_{1} e_{1}+\alpha_{2} e_{2}\right\rangle+\left\langle\left(Q^{*}\right)^{-1} f, D^{k} w\right\rangle .
$$

Assuming additionally that $f$ is a nonzero vector in $\mathcal{K}$ such that $\left(Q^{*}\right)^{-1} f \in \mathbb{C} e_{1} \oplus \mathbb{C} e_{2}$ and $\left(Q^{*}\right)^{-1} f$ is orthogonal to $\alpha_{1} e_{1}+\alpha_{2} e_{2}$, it follows that

$$
\left\langle f, \pi\left(x^{k}\right) \phi\right\rangle=\overline{\lambda^{k}} \cdot\left\langle\left(Q^{*}\right)^{-1} f, \alpha_{1} e_{1}+\alpha_{2} e_{2}\right\rangle=0
$$

for all integers $k$. Thus, $(\langle f, \tau(y) \phi\rangle)_{y \in\langle x\rangle}$ is the zero sequence and this clearly contradicts the fact that the linear map $f \mapsto(\langle f, \pi(h) \phi\rangle)_{h \in\langle x\rangle}$ is assumed to be injective.

It is perhaps worth noting that the arguments in the proof of Proposition 4 also establish the following.

Proposition 5. Let $G$ be a finite group and let $\pi$ be a faithful unitary representation of $G$ acting in $\mathcal{H}$ for which there exists an element $x \in G$ such that the order of $\pi(x)$ is at least equal to the dimension of $\mathcal{H}$ and $\pi(x)$ has an eigenvalue occurring with multiplicity strictly greater than one. Then $\pi$ must be spark deficient.

3.2. Groups with non-trivial centers are irreducibly spark deficient. Recall that $G$ is a $p$-group if each element in $G$ has a power of $p$ as its order.

Proposition 6. Let $G$ be a non-abelian finite group. If $G$ has a non-trivial center then $G$ is irreducibly spark deficient. Moreover, the following groups are all irreducibly spark deficient: p-groups and nilpotent groups.

Proof. Let $\pi$ be a unitary irreducible representation of $G$ acting in some Hilbert space $\mathcal{H}$ such that $\operatorname{dim} \mathcal{H}>1$. Let $Z(G)$ be the center of $G$. By assumption, $Z(G)$ is a non-trivial subgroup of $G$. Next, appealing to Schur's lemma, for arbitrary $z \in Z(G), \pi(z)$ is a scalar multiple of the identity operator acting on $\mathcal{H}$. Moreover, given any nonzero vector $f \in \mathcal{H}$, the span of $\pi(Z(G)) f$ is a one-dimensional vector subspace of $\mathcal{H}$ and consequently, $\pi$ is spark deficient. The second claim of the result follows from the well-known fact that p-groups and (more generally) nilpotent groups have non-trivial centers.

Remark 7. For a unitary representation $\pi$ of a finite group, the following statements are not mutually exclusive. (a) $\pi$ is spark deficient and (b) $\pi$ has an orbit which properly contains a full spark frame. For instance, it is known that the (non unitary characters) irreducible representations of the finite Heisenberg group (which is a nilpotent group) enjoy both of these properties [12. In fact, in the setting of groups with non-trivial centers, the more general concept of projective representation is more appropriate. 
Proposition 8. Let $G=K \rtimes H$ (where $H$ is a non-trivial subgroup of the automorphism group of $K$ ) be a finite semi-direct product group equipped with the operation

$$
(n, h)(m, s)=(n h(m), h s) \text { for }(n, h),(m, s) \in K \times H .
$$

If the center of $K$ has a non-trivial element which is fixed by the action of $H$ then $G$ is irreducibly spark deficient.

Proof. Let $e$ be the identity element in $H$ and let $n$ be a non-trivial element of the center of $N$ which is fixed by the action of the automorphism group $H$. For arbitrary $(m, h) \in G$,

$$
(n, e)(m, h)\left(n^{-1}, e\right)=(n m, h)\left(n^{-1}, e\right)=\left(n m h\left(n^{-1}\right), h\right) .
$$

Since $h\left(n^{-1}\right)=h^{-1}(n)=n$, we obtain $(n, e)(m, h)\left(n^{-1}, e\right)=\left(n m n^{-1}, h\right)$. Now, since $n$ commutes with $m$, we have $(n, e)(m, h)\left(n^{-1}, e\right)=\left(m n n^{-1}, h\right)=(m, h)$. Consequently, $(n, e)$ is a non-trivial element of the center of $G$.

A straightforward application of Proposition 8 gives the following.

Corollary 9. Let $G=\mathbb{Z}_{N} \rtimes H$ where $H$ is a cyclic group generated by $h \in \mathbb{Z}_{N}$ such that $h, N$ are relatively prime and $h>1$. Then $G$ has a non-trivial center if and only if there exists a nonzero element $x$ in $\mathbb{Z}_{N}$ which is a zero divisor satisfying the equation $(h-1) x=0$. In other words, if $h$ does not act freely on all of the nonzero elements of $\mathbb{Z}_{N}$, then $G$ is necessarily irreducibly spark deficient.

Note that if $G=\mathbb{Z}_{N} \rtimes H, H$ is non-trivial and $N$ is prime, then $G$ has a trivial center. In fact, we will prove in the subsequent section that when $N$ is prime then $G=\mathbb{Z}_{N} \rtimes H$ is irreducibly full spark (see Theorem 10.)

\section{FulL-SPARK FRAMES ARISING FROM IRREDUCIBLE REPRESENTATIONS OF $\mathbb{Z}_{N} \rtimes H$}

4.1. A summary of our main results. While the preceding section focuses on conditions under which a group fails to be irreducibly full spark, we shall provide here, some positive results for a class of semi-direct product groups. Our main aim in this section is to establish the following.

Theorem 10. For every prime natural number $N$ and every subgroup $H<\mathbb{Z}_{N}^{\times}$, the semi-direct product group $\mathbb{Z}_{N} \rtimes H$ is irreducibly full spark.

The following shows that Theorem 10 generally fails when $N$ is assumed to be composite.

Theorem 11. Let $N$ be a composite number and let $H$ be a subgroup of the automorphism group of $\mathbb{Z}_{N}$ of order at least $p$, where $p$ is the smallest prime divisor of $N$. Then, $\mathbb{Z}_{N} \rtimes H$ is irreducibly spark deficient.

If on the other hand, $N=p^{n}$ where $p$ a prime, $|H|<p$ and no two elements of $H$ are congruent $\bmod p$, then there is an irreducible unitary representation that is full spark.

As we shall see in the proof, there are irreducible unitary representations of $\mathbb{Z}_{N} \rtimes H$ of maximal dimension $|H|$ that are full spark, when $N=p^{n},|H|<p$, and no two elements of $H$ are congruent $\bmod p$. However, the group $\mathbb{Z}_{N} \rtimes H$ is neither irreducibly full spark, nor spark deficient in this case, as there are other irreducible unitary representations that are spark deficient. 
4.2. A central sufficient condition for the full spark property. Let $T$ denote the cyclic shift operator in $\mathbb{C}^{N}$, i.e.

$$
T\left(x_{0}, \ldots, x_{N-1}\right)=\left(x_{N-1}, x_{0}, \ldots, x_{N-2}\right),
$$

for every vector $\left(x_{0}, \ldots, x_{N-1}\right) \in \mathbb{C}^{N}$; the powers of $T$ will also be called cyclic permutations. A map of the form

$$
\left(x_{0}, x_{1}, \ldots, x_{N-1}\right) \longmapsto\left(a_{0} x_{0}, \ldots, a_{N-1} x_{N-1}\right)
$$

is a diagonal operator, and is denoted by $\operatorname{diag}\left(a_{0}, \ldots, a_{N-1}\right)$; its matrix representation is

$$
\left(\begin{array}{cccc}
a_{0} & 0 & \ldots & 0 \\
0 & a_{1} & \ldots & 0 \\
\vdots & \vdots & \ddots & \vdots \\
0 & 0 & \ldots & a_{N-1}
\end{array}\right)
$$

We will usually index the rows of an $N \times M$ matrix with elements from the cyclic group $\mathbb{Z}_{N}$. In this setting, we will say that a submatrix consists of consecutive rows, if the corresponding elements of $\mathbb{Z}_{N}$ are consecutive (more precisely, they form an arithmetic progression with difference $1 \bmod N$ ). For example, the submatrix consisting of the top and bottom row of an $N \times M$ matrix consists of consecutive rows, according to this definition.

Let $\Phi=\left\{\varphi_{1}, \ldots, \varphi_{M}\right\} \subseteq \mathbb{C}^{N}$ be a finite frame with frame bounds $B \geq A>0$. We will usually identify $\Phi$ with the $N \times M$ matrix whose columns are the frame elements, i.e.

$$
\Phi=\left(\begin{array}{cccc}
\mid & \mid & & \mid \\
\varphi_{1} & \varphi_{2} & \ldots & \varphi_{M} \\
\mid & \mid & & \mid
\end{array}\right) .
$$

The transpose of such a matrix is the matrix representation of the analysis operator.

The following result is central to the proofs of our main results.

Theorem 12. Suppose that the $N \times M$ matrix

$$
\mathcal{M}=\left(\begin{array}{cccc}
m_{00} & m_{01} & \ldots & m_{0, M-1} \\
m_{10} & m_{11} & \ldots & m_{1, M-1} \\
\vdots & \vdots & \ddots & \vdots \\
m_{N-1,0} & m_{N-1,1} & \ldots & m_{N-1, M-1}
\end{array}\right)
$$

has the following property: every minor consisting of consecutive rows from $\mathcal{M}$ is nonzero. Then, there is an open dense subset $\mathcal{S} \subseteq \mathbb{C}^{N}$ such that for every $f \in \mathcal{S}$, the set

$$
\mathcal{M}_{\ell} T^{k} f, 0 \leq \ell \leq M-1,0 \leq k \leq N-1,
$$

has the full spark property, where $\mathcal{M}_{\ell}=\operatorname{diag}\left(m_{0 \ell}, m_{1 \ell}, \ldots, m_{N-1, \ell}\right)$.

4.2.1. Proof of Theorem 12. Let $f=\left(z_{0}, z_{1}, \ldots, z_{N-1}\right)$. The determinant of the $N \times N$ formed by $N$ column vectors of the form (in a fixed order) $\mathcal{M}_{\ell} T^{k} f$ is a homogeneous polynomial in the coordinates of $f$ of degree $N$. We will show that every such selection of $N$ vectors yields a nonzero polynomial; the conclusion then follows easily, as we can take $\mathcal{S}$ to be the subset of $\mathbb{C}^{N}$ where none of these (finitely many) polynomials vanish. The 
complement of $\mathcal{S}$ is a finite union of varieties (closed subsets), having Lebesgue measure zero.

For this purpose, let

$$
\left\{\left(k_{0}, \ell_{0}\right), \ldots,\left(k_{N-1}, \ell_{N-1}\right)\right\} \subseteq \mathbb{Z}_{N} \times\{0,1, \ldots, M-1\}
$$

and define

and

$$
\Lambda=\left\{\mathcal{M}_{\ell_{j}} T^{k_{j}}: 0 \leq j \leq N-1\right\}
$$

$$
D_{\Lambda}(f)=\left(\begin{array}{llll}
\mathcal{M}_{\ell_{0}} T^{k_{0}} f & \mathcal{M}_{\ell_{1}} T^{k_{1}} f & \ldots & \mathcal{M}_{\ell_{N-1}} T^{k_{N-1}} f
\end{array}\right),
$$

where all the $\mathcal{M}_{\ell_{j}} T^{k_{j}} f$ are column vectors. We rewrite this matrix as

$$
D_{\Lambda}(f)=\left(D_{\Lambda_{0}}(f)\left|D_{\Lambda_{1}}(f)\right| \cdots \mid D_{\Lambda_{N-1}}(f)\right),
$$

where $D_{\Lambda_{j}}(f)$ is the subset of columns of $D_{\Lambda}(f)$ for which $k_{i}=j$ holds, possibly by rearranging the columns of $D_{\Lambda}(f)$ (an operation that only changes the sign of $\operatorname{det}\left(D_{\Lambda}(f)\right)$ ). The determinant of $D_{\Lambda}(f)$ is up to a sign equal to a sum of products of minors of the $D_{\Lambda_{j}}(f)$ matrices. Suppose that $D_{\Lambda_{j}}(f)$ is a $N \times L_{j}$ matrix (i.e. it consists of $L_{j}$ columns). We also label the rows of $D_{\Lambda}(f)$ by $\{0,1, \ldots, N-1\}$, with 0 being the top row and $N-1$ being the bottom one. Next, we consider a partition $\left(A_{0}, A_{1}, \ldots, A_{N-1}\right)$ of $\{0,1, \ldots, N-1\}$ such that $\left|A_{j}\right|=L_{j}$, and denote by $D_{\Lambda_{j}}\left(A_{j}\right)(f)$ the $L_{j} \times L_{j}$ minor of $D_{\Lambda_{j}}(f)$ consisting of the rows labeled by $A_{j}$ (in increasing order). As noted above, $\operatorname{det}\left(D_{\Lambda}(f)\right)$ is then a sum of products of the form

$$
\pm \prod_{j=0}^{N-1} \operatorname{det}\left(D_{\Lambda_{j}}\left(A_{j}\right)(f)\right) \text {. }
$$

When we expand the determinants in (4.1), we obtain a monomial of degree $N$ in $z_{0}, \ldots, z_{N-1}$. The coefficient of this monomial in (4.1) is

$$
\pm \prod_{j=0}^{N-1} \operatorname{det}\left(\mathcal{M}\left(A_{j}, B_{j}\right)\right)
$$

where $\mathcal{M}\left(A_{j}, B_{j}\right)$ is the submatrix of $\mathcal{M}$ with rows from $A_{j}$ and columns from $B_{j} \subseteq$ $\{0,1, \ldots, M-1\}$. An element $\ell$ belongs to $B_{j}$ if and only if $\mathcal{M}_{\ell} T^{j} f$ is a column of $D_{\Lambda}(f)$, which is equivalent to $\mathcal{M}_{\ell} T^{j} \in \Lambda$.

It is evident that if we use consecutive rows from the matrix $\mathcal{M}$, the expression in (4.2) will be nonzero, by hypothesis. In particular, we will use

$$
\begin{aligned}
P_{0} & =\left\{0,1, \ldots, L_{0}-1\right\} \\
P_{1} & =\left\{L_{0}, L_{0}+1, \ldots, L_{0}+L_{1}-1\right\} \\
& \vdots \\
P_{N-1} & =\left\{L_{0}+\cdots+L_{N-2}, \ldots, N-1\right\} .
\end{aligned}
$$

Thus, the monomial that corresponds to (4.1) has a nonzero contribution to its coefficient in $\operatorname{det}\left(D_{\Lambda}(f)\right)$ for this particular partition of $\{0,1, \ldots, N-1\}$; this monomial will henceforth be called the consecutive index monomial, or CI for short. However, $\operatorname{det}\left(D_{\Lambda}(f)\right)$ is the sum of terms (4.1) where the $A_{j}$ run through the partitions of $\{0,1, \ldots, N-1\}$ with 
$\left|A_{j}\right|=L_{j}$; in order to find the coefficient of the CI monomial in $\operatorname{det}\left(D_{\Lambda}(f)\right)$, we have to identify all such partitions which yield this monomial in (4.1), and then sum all such contributions of the form (4.2).

As we shall see, the CI monomial appears uniquely, i.e. there is a unique partition $A_{0}, \ldots, A_{N-1}$ that gives the CI monomial; by hypothesis, the total contribution, that is, the coefficient in $\operatorname{det}\left(D_{\Lambda}(f)\right)$ must be nonzero, thus $\operatorname{det}\left(D_{\Lambda}(f)\right)$ is eventually a nonzero polynomial, as desired.

Permuting the coordinates of $f$ does not change the fact that $\operatorname{det}\left(D_{\Lambda}(f)\right)$ is a nonzero polynomial in the coordinates of $f$, namely $z_{0}, \ldots, z_{N-1}$. In other words, if

$$
\Lambda^{\prime}=\left\{\mathcal{M}_{\ell} T^{k-s}:(k, \ell) \in \Lambda\right\},
$$

and $D_{\Lambda^{\prime}}(f)$ is defined similarly, that is,

$$
D_{\Lambda^{\prime}}(f)=\left(\begin{array}{lllll}
\mathcal{M}_{\ell_{0}} T^{k_{0}-s} f & \mathcal{M}_{\ell_{1}} T^{k_{1}-s} f & \ldots & \mathcal{M}_{\ell_{N-1}} T^{k_{N-1}-s} f
\end{array}\right),
$$

then $\operatorname{det}\left(D_{\Lambda}(f)\right)$ is a nonzero polynomial in $z_{0}, \ldots, z_{N-1}$ if and only if $\operatorname{det}\left(D_{\Lambda^{\prime}}(f)\right)$ is, where $s \in \mathbb{Z}_{N}$; indeed, since

$$
\operatorname{det}\left(D_{\Lambda^{\prime}}(f)\right)=\operatorname{det}\left(D_{\Lambda}\left(T^{-s} f\right)\right) .
$$

A cyclic permutation of the coordinates results in a cyclic permutation of the $P_{j}$, i.e. $P_{j}^{\prime}=P_{j+s}$, where the indices are taken $\bmod N$. We define $L_{j}^{\prime}=\left|P_{j}^{\prime}\right|$, and claim that there is such a cyclic permutation, for which

(I) $L_{0}^{\prime}>0$.

(II) The sets $P_{j}^{\prime}-j$ consist of non-negative integers; here, we assume that all the $P_{j}^{\prime}$ are subsets of $\{0,1, \ldots, N-1\}$.

This follows directly from the following combinatorial lemma by Dvoretzky and Motzkin, the so-called Cycle Lemma [7] (this version here is actually the second part of Theorem 3 in [7]):

Lemma 13. Let $p_{1} p_{2} \cdots p_{m+n}$ be a sequence of $m$ values equal to +1 and $n$ values equal to -1 , such that $m \geq n$. Then, there are at least $m-n+1$ cyclic permutations of the form $q_{1} q_{2} \cdots q_{m+n}$, such that all partial sums

$$
q_{1}+\cdots+q_{j}
$$

are non-negative, for $1 \leq j \leq m+n$.

We define $K_{j}=L_{0}+\cdots+L_{j-1}\left(K_{0}=0\right)$, so that

$$
P_{j}=\left\{K_{j}, K_{j}+1, \ldots, K_{j+1}-1\right\} .
$$

The set $P_{j}-j$ is then

$$
\left\{K_{j}-j, K_{j}-j+1, \ldots, K_{j+1}-j-1\right\}
$$

The second condition above is thus equivalent to

$$
L_{0}+\cdots+L_{j-1} \geq j .
$$

Next, we construct the following sequence of \pm 1 based on the data given by $L_{0}, \ldots, L_{N-1}$, as follows. We begin with a string of $L_{0}+1$ 's, followed by a single -1 , then a string of $L_{1}$ +1 's, followed by a single $-1, \ldots$, followed by a string of $L_{N-1}+1$ 's, and finally ending 
with a -1 . Since $L_{0}+\cdots+L_{N-1}=N$, the number of +1 is equal to the number of -1 , therefore by the Cycle Lemma, there is a cyclic permutation, whose partial sums are all non-negative. We can always choose such a permutation to end with a -1 , i.e. it begins with a string of $L_{s}+1$ 's, for some $s$. We then put $L_{j}^{\prime}=L_{j+s}^{\prime}$ (here the indices are taken $\bmod N)$, so that $L_{0}^{\prime}=L_{s}^{\prime}$, and the conclusion of the Cycle Lemma then is equivalent to the second condition, that is

$$
L_{0}^{\prime}+\cdots+L_{j-1}^{\prime} \geq j
$$

for all $j$.

Without loss of generality, we will assume henceforth that conditions (II) and (II) are satisfied for $\Lambda$ itself. We recall that the CI monomial corresponds to the standard $P_{j}$ partition.

We note that any desired partition has the form $\sigma\left(P_{0}\right), \ldots, \sigma\left(P_{N-1}\right)$, where $P_{0}, \ldots, P_{N-1}$ is the above fixed partition, which gives the CI monomial, and $\sigma$ runs through a quotient subgroup of the permutation group $S_{N}$, namely $S_{N} / \Gamma$, where $\Gamma$ the subset of permutations that leave each $P_{j}$ invariant (i.e. $\sigma\left(P_{j}\right)=P_{j}$ for all $j$ ); we will call such permutations trivial.

Therefore, every partition of $\{0,1, \ldots, N-1\}$ into $A_{j}$ with $\left|A_{j}\right|=L_{j}$ is obtained through a permutation $\sigma \in S_{N} / \Gamma$, by setting $A_{j}=\sigma\left(P_{j}\right)$. With every such partition (or equivalently, element of $\left.S_{N} / \Gamma\right)$ we associate a discrete random variable $X_{\sigma}$ with values at $\{0,1, \ldots, N-1\}$ satisfying

$$
\mathbb{P}\left[X_{\sigma}=j\right]=\frac{a_{j}}{N},
$$

where $Z^{\sigma}=z_{0}^{a_{0}} z_{1}^{a_{1}} \ldots z_{N-1}^{a_{N-1}}$ is the monomial associated with the partition $A_{0}, \ldots, A_{N-1}$. We put $X=X_{\iota}$, where $\iota$ is the identity partition; in other words, $X$ is the random variable associated with the CI monomial, which will be denoted by $Z$. We will show that the CI monomial appears uniquely by showing that the identity is the unique minimizer of the quantity $\mathbb{E}\left[X_{\sigma}^{2}\right]$ in $S_{N} /$ [1].

To do so, define the function $g$ on $\{0,1, \ldots, N-1\}$ with the property

$$
g^{-1}(j)=P_{j}, \quad 0 \leq j \leq N-1 .
$$

Obviously, $g$ is increasing, and is constant when restricted to each of the $P_{j}$. Conditions (II) and (III) above imply that $n-g(n) \geq 0$, for all $n \in\{0, \ldots, N-1\}$. We note that the indices appearing at the CI monomial are exactly the integers $n-g(n)$, counting multiplicities. On the other hand, the indices appearing in the monomial $Z^{\sigma}$ form precisely the union of multisets (i.e. we count an integer multiple times, if it appears on many sets) $\sigma\left(P_{j}\right)-j$, where the latter are taken $\bmod N$. So, for $0 \leq n \leq N-1$, the corresponding index for $Z^{\sigma}$ is $\sigma(n)-g(n)$. Since it is less than $N$ in absolute value, this index as an integer in $\{0, \ldots, N-1\}$ must either be $\sigma(n)-g(n)$ or $\sigma(n)-g(n)+N$. We thus define

$$
\sigma^{\prime}(n)= \begin{cases}\sigma(n), & \text { if } \sigma(n)-g(n) \geq 0 \\ \sigma(n)+N, & \text { otherwise. }\end{cases}
$$

\footnotetext{
${ }^{1}$ We note that the remaining of the proof follows in almost identical fashion as the proof of Theorem 4.3 from [12; we include the full proof for completeness.
} 
Let $C_{1}$ be the set of $n$ for which $\sigma(n)-g(n) \geq 0$ holds, and $C_{2}$ its complement in $\{0, \ldots, N-1\}$. Define

$$
f:\{0, \ldots, N-1\} \longrightarrow \sigma\left(C_{1}\right) \cup\left(\sigma\left(C_{2}\right)+N\right)
$$

to be strictly increasing ( $f$ is obviously unique), so that $f(n) \geq n$, for all $n$. Since $\sigma\left(C_{1}\right) \cup\left(\sigma\left(C_{2}\right)+N\right)$ is also the range of $\sigma^{\prime}$, there must be a permutation $\tau$ of this set, such that

$$
\sigma^{\prime}(n)=\tau(f(n)), \quad 0 \leq n \leq N-1 .
$$

Now, since $f(n) \geq n$, we must have $f(n)-g(n) \geq n-g(n) \geq 0$ for $0 \leq n \leq N-1$, hence

$$
\mathbb{E}\left[X^{2}\right] \leq \frac{1}{N} \sum_{n=0}^{N-1}(f(n)-g(n))^{2} .
$$

On the other hand,

$$
\begin{aligned}
\mathbb{E}\left[X_{\sigma}^{2}\right]-\frac{1}{N} \sum_{n=0}^{N-1}(f(n)-g(n))^{2} & =\frac{1}{N} \sum_{n=0}^{N-1}\left(\sigma^{\prime}(n)-g(n)\right)^{2}-\frac{1}{N} \sum_{n=0}^{N-1}(f(n)-g(n))^{2} \\
& =\frac{1}{N} \sum_{n=0}^{N-1}(\tau(f(n))-g(n))^{2}-\frac{1}{N} \sum_{n=0}^{N-1}(f(n)-g(n))^{2} \\
& =\frac{2}{N} \sum_{n=0}^{N-1} f(n) g(n)-\frac{2}{N} \sum_{n=0}^{N-1} \tau(f(n)) g(n) \\
& \geq 0
\end{aligned}
$$

where the latter inequality is justified by the rearrangement inequality. Thus,

$$
\mathbb{E}\left[X^{2}\right] \leq \frac{1}{N} \sum_{n=0}^{N-1}(f(n)-g(n))^{2} \leq \mathbb{E}\left[X_{\sigma}^{2}\right],
$$

for all permutations $\sigma$. If $C_{2} \neq \varnothing$, then for some $n$, we have $f(n)-g(n)>n-g(n)$, yielding strict inequality between $\mathbb{E}\left[X^{2}\right]$ and $\mathbb{E}\left[X_{\sigma}^{2}\right]$. So, $\mathbb{E}\left[X^{2}\right]$ can only equal $\mathbb{E}\left[X_{\sigma}^{2}\right]$ when $C_{2}=\varnothing$, which implies that $f$ must be the identity on $\{0, \ldots, N-1\}$. It also implies that $\sigma^{\prime}(n)=\sigma(n)$ for all $n$, which yields

$$
\begin{aligned}
\mathbb{E}\left[X_{\sigma}^{2}\right]-\mathbb{E}\left[X^{2}\right] & =\frac{1}{N} \sum_{n=0}^{N-1}(\sigma(n)-g(n))^{2}-\frac{1}{N} \sum_{n=0}^{N-1}(n-g(n))^{2} \\
& =\frac{2}{N} \sum_{n=0}^{N-1} n g(n)-\frac{2}{N} \sum_{n=0}^{N-1} \sigma(n) g(n) \\
& \geq 0,
\end{aligned}
$$

again by the rearrangement inequality; we note that equality can only occur when $\sigma$ is trivial, i.e. it leaves every $P_{j}$ (the level sets of $g$ ) invariant. This clearly shows that the expected value of $X^{2}$ is a unique statistical property for the CI monomial with respect to the given partition. Therefore, the CI monomial appears uniquely, and has a nonzero coefficient by hypothesis in the expansion of $\operatorname{det}\left(D_{\Lambda}(f)\right)$, thus rendering the latter a nonzero polynomial in $z_{0}, \ldots, z_{N-1}$. The result now follows by observing that $\mathcal{M}_{\ell} T^{k} f$, 
$0 \leq \ell \leq M-1,0 \leq k \leq N-1$ produces a full spark frame as long as $f$ avoids the finitely many varieties defined by the zero sets of the polynomials $\operatorname{det}\left(D_{\Lambda}(f)\right)$.

4.2.2. A sufficient condition for tightness. Next, we will examine when the frame

$$
\left\{\mathcal{M}_{\ell} T^{k} f: 0 \leq \ell \leq M-1,0 \leq k \leq N-1\right\}
$$

is tight.

Theorem 14. Let $\mathcal{M}=\left(m_{k \ell}\right)$ be a $N \times M$ matrix, and denote

$$
\mathcal{M}_{\ell}=\operatorname{diag}\left(m_{0 \ell}, m_{1 \ell}, \ldots, m_{N-1, \ell}\right) .
$$

Then, for a nonzero $f \in \mathbb{C}^{N}$, the frame $\left\{\mathcal{M}_{\ell} T^{k} f: 0 \leq \ell \leq M-1,0 \leq k \leq N-1\right\}$ is tight if the columns of $\mathcal{M}$ form a tight frame.

Proof. It is known that the columns of a matrix $\mathcal{M}$ form a tight frame if and only if $\mathcal{M M}^{*}$ is a multiple of the identity. If $\mathcal{M}^{k}$ denotes the $k$ th row of $\mathcal{M}$, this is equivalent to the fact that the rows of $\mathcal{M}$ are orthogonal and have the same norm. Without loss of generality, we may assume $\left\|\mathcal{M}^{k}\right\|=1$ for all $k$. Let $\Phi$ be the $N \times M N$ matrix whose columns are $\mathcal{M}_{\ell} T^{k} f$. Denote by $\Phi^{k}$ the $k$ th row of $\Phi$. We have

$$
\left\|\Phi^{k}\right\|^{2}=\left\|\mathcal{M}^{k}\right\|^{2}\|f\|^{2}=\|f\|^{2}
$$

and

$$
\left\langle\Phi^{j}, \Phi^{k}\right\rangle=\left\langle\mathcal{M}^{j}, \mathcal{M}^{k}\right\rangle \sum_{m \in \mathbb{Z}_{N}}\left\langle f, T^{m} f\right\rangle=0
$$

as desired.

4.3. A short overview of the theory of Mackey. In order to present the proof of Theorem 10, we need to describe (up to unitary equivalence) the set of all irreducible representations of the semi-direct product group $\mathbb{Z}_{N} \rtimes H$. To this end, we will make use of Mackey's analysis [9, Chapter 6].

Let $G=A H$ be a finite group where $A, H$ are subgroups, $A$ is normal in $G$, abelian and $A \cap H=\{e\}$. In other words, $G$ is isomorphic to the semi-direct product group $A \rtimes H$. Since $A$ is abelian, its unitary irreducible representations are all homomorphisms from $A$ into the torus group.

Let $\widehat{A}$ be the unitary dual of $A$. That is, the collection of all non-equivalent irreducible representations of $A$. The conjugation action of $G$ on $A$ induces an action on $\widehat{A}$ defined as follows. For $g \in G, \chi \in \widehat{A}$ and $x \in A$,

$$
[g \cdot \chi](x)=\chi\left(g^{-1} x g\right) \text {. }
$$

Let $\Sigma \subseteq \widehat{A}$ be a transversal for the $G$-orbits in $\widehat{A}$. For each $\chi \in \Sigma$, let $H_{\chi}$ be the stabilizer of $\chi$ in $H$. That is,

$$
H_{\chi}=\{h \in H: h \cdot \chi=\chi\}
$$

Put

$$
G_{\chi}=A H_{\chi}
$$


The map $g G_{\chi} \mapsto g \cdot \chi$ defines a bijection between $G / G_{\chi}$ and the orbit of $\chi$. Moreover, $G_{\chi}$ is the stabilizer of $\chi$ in $G$, and each unitary character $\chi$ can be extended to a unitary representation $\omega_{\chi}$ of $G_{\chi}$ as follows. Given $n \in A$ and $h \in H_{\chi}$,

$$
\omega_{\chi}(n h)=\chi(n) .
$$

Indeed, for $n, m \in A$ and $h, k \in H_{\chi}$, we verify that $\omega_{\chi}((n h)(m k))=\omega_{\chi}(n h) \omega_{\chi}(m k)$ as follows

$$
\begin{aligned}
\omega_{\chi}(n h m k) & =\omega_{\chi}\left(\left(n h m h^{-1}\right) h k\right) \\
& =\chi\left(n h m h^{-1}\right) \\
& =\chi(n) \chi\left(h m h^{-1}\right) \\
& =\chi(n)\left[h^{-1} \cdot \chi\right](m) .
\end{aligned}
$$

Since $h^{-1} \cdot \chi=\chi$

$$
\omega_{\chi}(n h m k)=\chi(n) \chi(m)=\omega_{\chi}(n h) \omega_{\chi}(m k) .
$$

Next, given a unitary irreducible representation $\rho$ of $H_{\chi}$ acting in $\mathcal{H}_{\rho}$, the tensor representation $\omega_{\chi} \otimes \rho$ is an irreducible representation of the subgroup $G_{\chi}$. Letting

$$
\pi_{\rho, \chi}=\operatorname{ind}_{G_{\chi}}^{G}\left(\omega_{\chi} \otimes \rho\right)
$$

be the representation obtained by inducing $\omega_{\chi} \otimes \rho$ from $G_{\chi}$ to $G$, according to the theory of Mackey, $\pi_{\rho, \chi}$ is necessarily irreducible [9, Chapter 6]. In fact, all irreducible representations of $G$ arise in this fashion. Precisely, for each $\chi \in \Sigma$, letting $\widehat{H_{\chi}}$ be a collection consisting of non-equivalent unitary irreducible representations of $H_{\chi}$, every unitary irreducible representation of $G=A H$ is equivalent to one element of the following set

$$
\bigcup_{\chi \in \Sigma}\left\{\pi_{\rho, \chi}: \rho \in \widehat{H_{\chi}}\right\} \text {. }
$$

Moreover, each $\pi_{\rho, \chi}$ is realized as acting in the space $\mathcal{F}_{\rho, \chi}$ consisting of functions $f: G \rightarrow$ $\mathcal{H}_{\rho}$ satisfying the covariance relation

$$
f(x z)=\left(\omega_{\chi} \otimes \rho\right)\left(z^{-1}\right) f(x) \text { where } z \in G_{\chi} .
$$

The vector space $\mathcal{F}_{\rho, \chi}$ is naturally endowed with the inner product

$$
\langle v, w\rangle_{\mathcal{F}_{\rho, \chi}}=\sum_{g H \in G / G_{\chi}}\langle v(g), w(g)\rangle_{\mathcal{H}_{\rho}} .
$$

Note that the covariance relation described in (4.6) implies that the inner product (4.7) is indeed well defined.

The action of $\pi_{\rho, \chi}$ is defined as follows. For $f \in \mathcal{F}_{\rho, \chi}$ and $g, z \in G$,

$$
\pi_{\rho, \chi}(g) f(z)=f\left(g^{-1} z\right) .
$$

Remark 15. Note that in cases where the subgroup $H_{\chi}$ is the singleton containing the identity in $H$, the extension representation $\omega_{\chi}$ is trivial in the sense that $\omega_{\chi}=\chi$. It is 
thus convenient to realize the action of $\operatorname{ind}_{A}^{G}(\chi)$ as acting in $l^{2}(H)$ as follows. Since $H \subseteq G$ is a transversal for $G / A$, given $f \in \mathcal{F}_{1, \chi}$, we have

$$
f(h a)=\chi\left(a^{-1}\right) f(h) \quad(a, h) \in A \times H .
$$

Therefore, $\mathcal{F}_{1, \chi}$ can be naturally identified with $l^{2}(H)$, and the following is immediate. For $f \in \mathcal{F}_{1, \chi}, a \in A$,

$$
\begin{aligned}
{\left[\pi_{1, \chi}(a) f\right](h) } & =\left[\operatorname{ind}_{A}^{G}(\chi)(a) f\right](h) \\
& =f\left(a^{-1} h\right) \\
& =f\left(h h^{-1} a^{-1} h\right) \\
& =\chi\left(h^{-1} a h\right) f(h)
\end{aligned}
$$

and for $k \in H$,

$$
\left[\pi_{1, \chi}(k) f\right](h)=\left[\operatorname{ind}_{A}^{G}(\chi)(k) f\right](h)=f\left(k^{-1} h\right) .
$$

In summary, elements of the normal part of the group act diagonally, while the restriction of $\pi_{1, \chi}$ to $H$ is unitarily equivalent to the left regular representation of $H$.

4.4. Proof of Theorem 10. Let $\pi$ be an irreducible and unitary representation of $\mathbb{Z}_{N} \rtimes$ $H$ acting in a Hilbert space $\mathcal{H}_{\pi}$. Since $\pi$ is irreducible [19, Theorem 3] it is known that for any nonzero vector $f \in \mathcal{H}_{\pi},\{\pi(g) f: g \in G\}$ is a tight frame for $\mathcal{H}_{\pi}$.

To prove Theorem 10, it is enough to show that there exists a nonzero vector whose orbit is a full spark frame in $\mathcal{H}_{\pi}$. Since the case where $\pi$ is a unitary character is trivially true, we shall not address it, and we will instead focus on representations of dimensions strictly larger than one.

Since $N$ is assumed to be prime, the automorphism group of $\mathbb{Z}_{N}=\{0,1, \cdots, N-1\}$ is the multiplicative modular group $\mathbb{Z}_{N}^{\times}=\{1, \cdots, N-1\}$ which is isomorphic to $\mathbb{Z}_{N-1}$.

Let $G=\mathbb{Z}_{N} \rtimes \mathbb{Z}_{N}^{\times}$be the semi-direct product group endowed with the following multiplication law. For $x, y \in \mathbb{Z}_{N}=\{0,1, \cdots, N-1\}$ and $a, b \in \mathbb{Z}_{N}^{\times}=\{1, \cdots, N-1\}$,

$$
(x, a)(y, b)=(x+a y, a b) \text {. }
$$

For a unitary character,

$$
\chi_{\xi}: x \mapsto \exp \left(\frac{2 \pi i \xi x}{N}\right) \quad\left(\xi \in \mathbb{Z}_{N}\right)
$$

of $\mathbb{Z}_{N}$, and $a \in \mathbb{Z}_{N}^{\times}$, it is easy to verify that

$$
a \cdot \chi_{\xi}=\chi_{a^{-1} \xi}
$$

Under the action of $G$, the unitary dual of $\mathbb{Z}_{N}$ is partitioned into two classes of orbits:

$$
\left\{\chi_{0}\right\} \cup\left\{\chi_{1}, \ldots, \chi_{N-1}\right\} .
$$

$\Sigma=\left\{\chi_{0}, \chi_{1}\right\}$ is a transversal for the orbits of $G$ in the unitary dual of $\mathbb{Z}_{N}$. Moreover, the stabilizer subgroup of $\chi_{0}$ coincides with $G$ while the stabilizer subgroup corresponding to the representation $\chi_{1}$ is trivial. Since $\mathbb{Z}_{N}^{\times}$is abelian, it follows from Mackey's analysis that up to unitary equivalence, the unitary dual of $G$ is parametrized by the set

$$
\left\{\omega_{\chi_{0}} \otimes \rho: \rho \in \widehat{\mathbb{Z}}_{N}^{\times}\right\} \cup\left\{\pi=\operatorname{ind}_{\mathbb{Z}_{N} \rtimes\{(0,1)\}}^{G}\left(\chi_{1}\right)\right\} .
$$


On the one hand, all representations in $\left\{\omega_{\chi_{0}} \otimes \rho: \rho \in \widehat{\mathbb{Z}}_{N}^{\times}\right\}$are characters, and there are $(N-1)$-many of them. On the other, every irreducible representation of $G$ of dimension larger than one is unitarily equivalent to $\pi=\operatorname{ind}_{\mathbb{Z}_{N} \rtimes\{(0,1)\}}^{G}\left(\chi_{1}\right)$. The representation $\pi$ is realized as acting in $l^{2}\left(\mathbb{Z}_{N}^{\times}\right)$as follows. Letting a be a generator of the multiplicative group $\mathbb{Z}_{N}^{\times}$and $\omega=\exp \left(\frac{2 \pi i}{N}\right)$,

$$
\pi(\gamma) f\left(a^{j}\right)=\left\{\begin{array}{c}
\omega^{a^{-j} x} f\left(a^{j}\right) \text { if } \gamma=(x, 1) \\
f\left(a^{j-\kappa}\right) \text { if } \gamma=\left(0, a^{\kappa}\right)
\end{array}\right.
$$

for $f \in l^{2}\left(\mathbb{Z}_{N}^{\times}\right)=\mathbb{C}^{N-1}$. Next, the matrix $\mathcal{M}$ defined in Theorem 12 takes the form $(4.10)$

$$
\mathcal{M}=\mathcal{M}_{N}(\omega)=\left(\begin{array}{ccccc}
1 & \omega & \omega^{2} & \cdots & \omega^{(N-1)} \\
1 & \omega^{a^{-1}} & \omega^{2 a^{-1}} & \cdots & \omega^{(N-1) a^{-1}} \\
1 & \omega^{a^{-2}} & \omega^{2 a^{-2}} & \cdots & \omega^{(N-1) a^{-2}} \\
\vdots & \vdots & \vdots & \ddots & \vdots \\
1 & \omega^{a^{-(N-2)}} & \omega^{2 a^{-(N-2)}} & \cdots & \omega^{(N-1) a^{-(N-2)}}
\end{array}\right)=\left(\omega^{k a^{-j}}\right)_{0 \leq j \leq N-2,0 \leq k \leq N-1} .
$$

Precisely, $\mathcal{M}$ is an $(N-1) \times N$ matrix obtained by arranging the diagonals of $\pi(x, 1), x \in$ $\mathbb{Z}_{N}$ in columns.

Suppose on the other hand that $H$ is a proper subgroup of $\mathbb{Z}_{N}^{\times}$. Clearly, the order of $H$ must strictly divide $N-1$. Let $\Gamma=\mathbb{Z}_{N} \rtimes H$ be the semi-direct product group with a multiplication law defined similarly to (4.8). Note that since $N$ is a prime number, every nonzero element of $\mathbb{Z}_{N}$ is a non-zero divisor and as such, the stabilizer subgroup of $\chi_{\xi}$ for $\xi \in \mathbb{Z}_{N} \backslash\{0\}$ is the trivial subgroup of $H$. In other words, if $\pi$ is an irreducible representation of $\Gamma$ which is not a character, according to the theory of Mackey, there exists $\xi \in \mathbb{Z}_{N} \backslash\{0\}$, such that $\pi$ is unitarily equivalent to a representation induced by the unitary character

$$
\chi_{\xi}: \mathbb{Z}_{N} \ni x \mapsto \exp \left(\frac{2 \pi i \xi x}{N}\right) \quad\left(\xi \in \mathbb{Z}_{N} \backslash\{0\}\right) .
$$

Such a representation may be modeled as acting in $l^{2}(H)$ as follows:

$$
\pi_{\xi}(x, s) f(h)=\omega_{\xi}^{h^{-1} x} f\left(s^{-1} h\right)
$$

for $\omega_{\xi}=\exp \left(\frac{2 \pi \xi i}{N}\right)$.

It is perhaps worth noting that if $\pi$ is an irreducible representation of $\mathbb{Z}_{N} \rtimes \mathbb{Z}_{N}^{\times}$acting on $\mathcal{H}_{\pi}$ and if additionally, $\pi$ is not a character, then

$$
\operatorname{dim}\left(\mathcal{H}_{\pi}\right)=N-1>\operatorname{dim}\left(l^{2}(H)\right)=|H| .
$$

This observation implies that the restriction of an irreducible representation of $\mathbb{Z}_{N} \rtimes \mathbb{Z}_{N}^{\times}$ which is not a character to its subgroup $\Gamma$ can never be equivalent to an irreducible representation of $\Gamma$. In any case, the matrix $\mathcal{M}$ as defined in Theorem 12 takes the form

$$
\left(\omega_{\xi}^{k a^{-j}}\right)_{0 \leq j \leq|H|-1,0 \leq k \leq N-1}
$$

In either case any square submatrix of $\mathcal{M}$ satisfies the sufficient conditions described in the following result which was established in [8, Theorem 6]. 
Proposition 16. Let $N$ be a prime number and let $\omega$ be a $N$-th root of unity. Suppose that $a_{1}, \cdots, a_{n} \in \mathbb{Z}$ are pairwise incongruent modulo $N$. Assuming the same for $b_{1}, \cdots, b_{n} \in$ $\mathbb{Z}$, the matrix $\left(\omega^{a_{j} b_{k}}\right)_{1 \leq k, j \leq n}$ is invertible.

Appealing to Theorem [12, we conclude that if $H$ is any subgroup of the automorphism group of $\mathbb{Z}_{N}, N$ is a prime natural number, and $\pi$ is a unitary irreducible representation of $\mathbb{Z}_{N} \rtimes H$ acting in some Hilbert space $\mathbb{C}^{M}$, then for almost every vector $f \in \mathbb{C}^{M}$ (with respect to the Lebesgue measure on $\mathbb{C}^{M}$ ) the orbit of $f$ is an equal norm tight frame which is also full spark.

Example 17. Let $G=\mathbb{Z}_{5} \rtimes \mathbb{Z}_{4}=\{0,1,2,3,4\} \rtimes\{1,2,3,4\}$. Appealing to Mackey's analysis, the unitary dual of $G$ contains (up to equivalence) four unitary characters and one irreducible representation $\pi$ acting in $\mathbb{C}^{4} . \pi=\operatorname{ind}_{\mathbb{Z}_{5} \rtimes\{1\}}^{G}\left(\chi_{1}\right)$ is realized as acting in $\mathbb{C}^{4}$ as follows:

$$
\pi(0,2)=T=\left(\begin{array}{llll}
0 & 1 & 0 & 0 \\
0 & 0 & 1 & 0 \\
0 & 0 & 0 & 1 \\
1 & 0 & 0 & 0
\end{array}\right) \text { and } \pi(1,1)=D=\left(\begin{array}{cccc}
e^{\frac{2 \pi i}{5}} & 0 & 0 & 0 \\
0 & e^{\frac{2 \pi i 3}{5}} & 0 & 0 \\
0 & 0 & e^{\frac{2 \pi i 3^{2}}{5}} & 0 \\
0 & 0 & 0 & e^{\frac{2 \pi i 3^{3}}{5}}
\end{array}\right)
$$

According to Theorem 10, since 5 is a prime number, we know that $\pi$ is full spark. To construct a fullspark frame, we proceed as follows. Let $v \in \mathbb{C}^{4}$ such that $v_{k}=t^{k^{2}}$ for $k \in\{0, \cdots, 3\}$. The matrix representation of the corresponding analysis operator is of the form $C=\left(\frac{A}{B}\right)$ where

$$
A=\left(\begin{array}{cccc}
1 & t & t^{4} & t^{9} \\
t & t^{4} & t^{9} & 1 \\
t^{4} & t^{9} & 1 & t \\
t^{9} & 1 & t & t^{4} \\
\omega & t \omega^{3} & t^{4} \omega^{4} & t^{9} \omega^{2} \\
t \omega & t^{4} \omega^{3} & t^{9} \omega^{4} & \omega^{2} \\
t^{4} \omega & t^{9} \omega^{3} & \omega^{4} & t \omega^{2} \\
t^{9} \omega & \omega^{3} & t \omega^{4} & t^{4} \omega^{2} \\
\omega^{2} & t \omega^{6} & t^{4} \omega^{8} & t^{9} \omega^{4} \\
t \omega^{2} & t^{4} \omega^{6} & t^{9} \omega^{8} & \omega^{4}
\end{array}\right), B=\left(\begin{array}{cccc}
t^{4} \omega^{2} & t^{9} \omega^{6} & \omega^{8} & t \omega^{4} \\
t^{9} \omega^{2} & \omega^{6} & t \omega^{8} & t^{4} \omega^{4} \\
\omega^{3} & t \omega^{9} & t^{4} \omega^{12} & t^{9} \omega^{6} \\
t \omega^{3} & t^{4} \omega^{9} & t^{9} \omega^{12} & \omega^{6} \\
t^{4} \omega^{3} & t^{9} \omega^{9} & \omega^{12} & t \omega^{6} \\
t^{9} \omega^{3} & \omega^{9} & t \omega^{12} & t^{4} \omega^{6} \\
\omega^{4} & t \omega^{12} & t^{4} \omega^{16} & t^{9} \omega^{8} \\
t \omega^{4} & t^{4} \omega^{12} & t^{9} \omega^{16} & \omega^{8} \\
t^{4} \omega^{4} & t^{9} \omega^{12} & \omega^{16} & t \omega^{8} \\
t^{9} \omega^{4} & \omega^{12} & t \omega^{16} & t^{4} \omega^{8}
\end{array}\right)
$$

and $\omega=e^{\frac{2 \pi i}{5}}$. Letting $t$ be any transcendental complex number over the field $\mathbb{Q}(\omega)$, the orbit of $v$ is a full spark and tight frame consisting of twenty vectors.

4.5. Proof of Theorem 11. Let $N$ be a composite number and let $H$ be a subgroup of the automorphism group of $\mathbb{Z}_{N}$ of order at least $p$, where $p$ is the smallest prime factor of $N$. We first consider the unitary irreducible representations of $\mathbb{Z}_{N} \rtimes H$ that are induced from characters of $\widehat{\mathbb{Z}_{N}}$ of maximal order (see subsection 4.3, especially the Remark towards the end). Such a character has the form $\chi_{m}$ defined by

$$
\chi_{m}: x \mapsto \exp \left(\frac{2 \pi i m x}{N}\right)
$$


where $\operatorname{gcd}(m, N)=1$, and its stabilizer in $G=\mathbb{Z}_{N} \rtimes H$ is $G_{1}=\mathbb{Z}_{N} \rtimes\{1\}$. Appealing to Mackey's analysis, the representation

$$
\pi_{m}=\operatorname{ind}_{G_{1}}^{G} \chi_{m}
$$

is an irreducible representation acting on a space $\mathcal{H}_{m}$ of dimension $|H| \geq p$. Actually, $\mathcal{H}_{m}$ is the direct sum of one-dimensional spaces $W_{h}=(0, h) W_{1}$, indexed by $h \in H$. A generic element $v$ of $\mathcal{H}_{m}$ is then written as

$$
v=\sum_{h \in H}(0, h) w_{h}
$$

where $w_{h} \in W_{1}=W$, where $W$ is the one-dimensional space on which $\chi_{m}$ acts. Obviously, $\{0\} \rtimes H$ acts on $\mathcal{H}_{m}$ by permuting coordinates (i.e. $\pi_{m} \mid H$ is just the regular representation of $H$ ). We only need to determine the action of the other factor, namely $\mathbb{Z}_{N} \rtimes\{1\}$. For arbitrary $x \in \mathbb{Z}_{N}$, we obtain

$$
(x, 1) v=\sum_{h \in H}(x, h) w_{h}=\sum_{h \in H}(0, h)\left(h^{-1} x, 1\right) w_{h}=\sum_{h \in H} \exp \left(\frac{2 \pi i m h^{-1} x}{N}\right)(0, h) w_{h} .
$$

Hence $\mathbb{Z}_{N} \rtimes\{1\}$ acts diagonally on $\mathcal{H}_{m}$. The orbit of $v \in \mathcal{H}_{m}$ under the action of $\mathbb{Z}_{N} \rtimes\{1\}$ is the set of vectors that appear as columns of the $\mathcal{M}^{\top} v$, where $\mathcal{M}$ is the $|H| \times N$ submatrix of the Fourier matrix of $\mathbb{Z}_{N}$, whose rows are indexed by $m \cdot H$. If the columns of $\mathcal{M}$ form a spark deficient set, then $\mathcal{M}^{\top} v$ is also spark deficient for every choice of $v$, proving Theorem 11.

For this purpose, we invoke the following definition and theorem by Alexeev, Cahill, and Mixon [1].

Definition 4.1. [1, Definition 7] We say that a subset $\mathcal{S} \subseteq \mathbb{Z}_{N}$ is uniformly distributed over the divisors of $N$ if, for every divisor $d$ of $N$, the $d$ cosets of $\langle d\rangle$ partition $\mathcal{S}$ into subsets, each of size $\left\lfloor\frac{|\mathcal{S}|}{d}\right\rfloor$ or $\left\lceil\frac{|\mathcal{S}|}{d}\right\rceil$.

Theorem 18. [1, Theorem 9] Select rows indexed by $\mathcal{S} \subseteq \mathbb{Z}_{N}$ from the $N \times N$ discrete Fourier transform matrix to build the submatrix $F$. If $F$ is full spark, then $\mathcal{S}$ is uniformly distributed over the divisors of $N$. If in addition, $N$ is a prime power, then the converse holds as well: if $\mathcal{S}$ is uniformly distributed over the divisors of $N$, then $F$ is full spark.

We conclude the proof of Theorem 11 simply by noting that if $p$ is the smallest prime factor of $N$ and $H<\mathbb{Z}_{N}^{\times}$is a subgroup of order at least $p$, then $m \cdot H$ cannot be uniformly distributed over the divisors of $N$ for $\operatorname{gcd}(m, N)=1$, as it cannot be uniformly distributed $\bmod p$, since no element in $H$ can be a multiple of $p$, hence

$$
0=\left|H \cap p \mathbb{Z}_{N}\right|<1 \leq\left\lfloor\frac{|H|}{p}\right\rfloor,
$$

thus the columns of $\mathcal{M}$ form a spark deficient set, as desired.

Regarding the unitary irreducible representations of $\mathbb{Z}_{N} \rtimes H$ which arise from $\chi_{m}$, where $\operatorname{gcd}(m, N)=d>1$, we simply observe that $\chi_{m}$ is not faithful, therefore, all the corresponding representations of $\mathbb{Z}_{N} \rtimes H$ that are obtained through Mackey's method, are not faithful, hence spark deficient, proving the first part of the Theorem. We remark 
that if we mod out the kernel, we obtain a faithful irreducible representation of $\mathbb{Z}_{N / d} \rtimes H$, that arises from the character

$$
\psi_{m}: \mathbb{Z}_{N / d} \ni x \mapsto \exp \left(\frac{2 \pi i m x}{N}\right) .
$$

For the second part of Theorem 11, we consider again the characters $\chi_{m}$ of $\mathbb{Z}_{N}$ with $\operatorname{gcd}(m, N)=1$ and we apply the reverse direction of Theorem 18 for prime powers, as any minor of the resulting matrix $\mathcal{M}$ will consist of rows indexed by a subset of $\mathbb{Z}_{N}$ which has fewer than $p$ elements, no two of which are congruent $\bmod p$. By definition, this is an equidistributed set with respect to any divisor of $p^{n}$, therefore, any minor of $\mathcal{M}$ is nonzero; the proof then follows immediately by Theorem [12, concluding the proof of Theorem 11.

Remark 19. We notice that the full spark representation of $\mathbb{Z}_{N} \rtimes H$ mentioned in the proof has dimension $|H|$, where $N=p^{n}$ a prime power and $|H|<p$. Furthermore, the subgroup $H$ must have distinct elements $\bmod p$; this forces $H$ to be a subgroup of the unique subgroup of $\mathbb{Z}_{N}^{\times}$of order dividing $p-1$. Therefore, for any $d$ and a prime $p \equiv 1 \bmod d$, there is a full spark equal norm tight frame in $\mathbb{C}^{d}$ consisting of $d p^{n}$ elements, for every positive integer $n$.

Corollary 20. Let $N$ be even and $H$ be a subgroup of $\mathbb{Z}_{N}^{\times}$. Define the irreducible representation $\pi$ of $G=\mathbb{Z}_{N} \rtimes H$ as above, acting on $\mathcal{H}_{\pi}$. Then, the set $\{\pi(g) v\}_{g \in G}$ is spark deficient for every $v \in \mathcal{H}_{\pi}$. If $N$ is odd and $H=\{1,-1\}$, then there exist vectors $v \in \mathcal{H}_{\pi}$, such that $\{\pi(g) v\}_{g \in G}$ is full spark.

Proof. The first part is an immediate consequence of Theorem 18, while the second part follows from Theorem 12 ,

In the general case where $N$ is composite, the representation $\pi$ can only be full spark if $|H|<p$, where $p$ is the smallest prime factor of $N$, and $H$ does not have two elements congruent $\bmod q$, for any prime factor $q$ of $N$. This, in particular, means that the natural $\bmod q$ reduction $H \rightarrow \mathbb{Z}_{q}^{\times}$is injective, showing that $H$ must be cyclic. This proves:

Corollary 21. With notation as above, if $H<\mathbb{Z}_{N}^{\times}$is non-cyclic, then $\pi$ is spark deficient.

Remark 22. It is known that the automorphism group of $\mathbb{Z}_{N}$ is cyclic if and only if $N=1,2,4, p^{k}, 2 p^{k}$ where $p$ is some positive odd prime number. In light of the preceding result, it is then clear that for any other values of $N$, any irreducible representation of $\mathbb{Z}_{N} \rtimes \mathbb{Z}_{N}^{\times}$obtained by inducing a unitary character of the normal group $\mathbb{Z}_{N}$ is spark deficient.

\section{A generalization of the CASE of $\mathbb{Z}_{N} \rtimes H$ Without AN UNDERLying Group STRUCTURE}

Our primary objective in this section is to generalize Theorem 10. Let 


$$
T=\left(\begin{array}{ccccc}
0 & 1 & 0 & \cdots & 0 \\
0 & 0 & 1 & \cdots & \vdots \\
\vdots & \vdots & 0 & \ddots & 0 \\
0 & \cdots & 0 & \ddots & 1 \\
1 & 0 & 0 & \cdots & 0
\end{array}\right)
$$

and fix $\left(\xi_{0}, \xi_{1}, \cdots, \xi_{N-1}\right) \in \mathbb{Z}^{N}$ such that

$$
0 \leq \xi_{0}<\xi_{1}<\cdots<\xi_{N-1}
$$

For a real number $\lambda$, define

$$
D_{\lambda}=\left(\begin{array}{cccc}
e^{2 \pi i \lambda \xi_{0}} & 0 & \cdots & 0 \\
0 & e^{2 \pi i \lambda \xi_{1}} & \cdots & 0 \\
\vdots & \vdots & \ddots & \vdots \\
0 & 0 & \cdots & e^{2 \pi i \lambda \xi_{N-1}}
\end{array}\right)
$$

Next, let $\left\{D_{\lambda} T^{\kappa}: \lambda \in \Lambda_{\tau}, \kappa \in \mathbb{Z}_{N}\right\}$ be a collection of matrices unitary matrices where

$$
\Lambda_{\tau}=\left\{0 \leq \tau \lambda_{0}<\tau \lambda_{1}<\cdots<\tau \lambda_{M-1}\right\}
$$

is a finite subset of the real line, $\tau$ is a fixed real number and $\lambda_{0}, \cdots, \lambda_{M-1}$ are some positive integers. We note that the collection of matrices $\left\{D_{\lambda} T^{\kappa}: \lambda \in \Lambda_{\tau}, \kappa \in \mathbb{Z}_{N}\right\}$ rarely has the structure of a group.

Remark 23. For the specific case where $\left(\xi_{0}, \xi_{1}, \cdots, \xi_{N-1}\right)=(0,1, \cdots, N-1)$ and $\lambda=$ $\frac{1}{N}$, we obtain

$$
D_{\frac{1}{N}}=\left(\begin{array}{cccc}
e^{\frac{2 \pi i(0)}{N}} & 0 & \cdots & 0 \\
0 & e^{\frac{2 \pi i}{N}} & \cdots & 0 \\
\vdots & \vdots & \ddots & \vdots \\
0 & 0 & \cdots & e^{\frac{2 \pi i(N-1)}{N}}
\end{array}\right)
$$

Under these assumptions, it is quite easy to verify that the group generated by $D_{\frac{1}{N}}, T$ is the discrete Heisenberg group. Moreover, the group $\left\langle D_{\frac{1}{N}}, T\right\rangle$ acts unitarily and irreducibly on $\mathbb{C}^{N}$ and it is known that there exists a vector $f \in \mathbb{C}^{N}$ such that $\left\{D_{\frac{1}{N}}^{m} T^{k} f:(m, k) \in \mathbb{Z}_{N} \times \mathbb{Z}_{N}\right\}$ is a fullspark frame for $\mathbb{C}^{N}$.

We shall establish the following result.

Theorem 24. If $e^{2 \pi i \tau}$ is either transcendental or algebraic over the rationals with sufficiently large degree, then there exists a nonzero vector $f \in \mathbb{C}^{N}$ such that the collection $\left\{D_{\lambda} T^{\kappa} f: \lambda \in \Lambda_{\tau}, \kappa \in \mathbb{Z}_{N}\right\}$ is a full spark frame for $\mathbb{C}^{N}$. 
The matrix $\mathcal{M}$ as defined in Theorem 12 takes the form

$$
\mathcal{M}:=\mathcal{M}_{\tau}=\left(\begin{array}{cccc}
e^{2 \pi i\left(\tau \lambda_{0}\right) \xi_{0}} & e^{2 \pi i\left(\tau \lambda_{1}\right) \xi_{0}} & \cdots & e^{2 \pi i\left(\tau \lambda_{M-1}\right) \xi_{0}} \\
e^{2 \pi i\left(\tau \lambda_{0}\right) \xi_{1}} & e^{2 \pi i\left(\tau \lambda_{1}\right) \xi_{1}} & \cdots & e^{2 \pi i\left(\tau \lambda_{M-1}\right) \xi_{1}} \\
\vdots & \vdots & \ddots & \vdots \\
e^{2 \pi i\left(\tau \lambda_{0}\right) \xi_{N-1}} & e^{2 \pi i\left(\tau \lambda_{1}\right) \xi_{N-1}} & \cdots & e^{2 \pi i\left(\tau \lambda_{M-1}\right) \xi_{N-1}}
\end{array}\right)
$$

To prove Theorem 24, we will need some additional tools. A Young diagram is a collection of cells arranged in the form of left-justified and weakly decreasing number of cells in each row. By listing the number of cells in each row, we obtain a partition of a fixed positive integer which represents the total number of cells in the diagram. A partition $\kappa=\left(\kappa_{1}, \cdots, \kappa_{m}\right)$ is a finite sequence of weakly decreasing positive integers, and a Young tableau is a filling that is (a) weakly increasing across each row and (b) strictly increasing down each column. A Young tableau obtained by filling a Young diagram associated with a partition $\kappa$ is called a tableau of shape $\kappa$.

For each partition $\kappa$, there is a corresponding symmetric polynomial known as a Schur polynomial described as follows. Given any filling $\mu$ of a Young diagram, we define a monomial $x^{\mu}$ which is obtained by taking the product of the variables $x_{k}$ corresponding to the indices $k$ appearing in $\mu$. More precisely, such a monomial takes the form

$$
x^{\mu}=\prod_{k=1}^{m} x_{k}^{\text {number of times } k \text { occurs in } \mu} .
$$

The Schur polynomial

$$
s_{\kappa}\left(x_{1}, x_{2}, \cdots, x_{m}\right)=\sum x^{\mu}
$$

is obtained by adding of all such monomials coming from tableaux $\mu$ of shape $\kappa$ using the natural numbers $1, \cdots, m$.

5.1. Proof of Theorem 24. In light of Theorem 12, to establish the first part of the result, it is enough to show that if the complex number $e^{2 \pi i \tau}$ is transcendental or algebraic over the rationals with a sufficiently large degree of extension, then every minor of $\mathcal{M}$ is nonzero. Let $S$ be a submatrix of $\mathcal{M}$ of order $L$. There exists an elementary (permutation) matrix $E$ of order $n$ such that

$$
E S=\left(e^{2 \pi i \tau \lambda_{s_{k}} \xi_{r_{j}}}\right)_{0 \leq j \leq L-1,0 \leq k \leq L-1}=\left(\begin{array}{cccc}
e^{2 \pi i \tau \lambda_{s_{0}} \xi_{r_{0}}} & e^{2 \pi i \tau \lambda_{s_{1}} \xi_{r_{0}}} & \cdots & e^{2 \pi i \tau \lambda_{s_{L-1}} \xi_{r_{0}}} \\
e^{2 \pi i \tau \lambda_{s_{0}} \xi_{r_{1}}} & e^{2 \pi i \tau \lambda_{s_{1}} \xi_{r_{1}}} & \cdots & e^{2 \pi i \tau \lambda_{s_{L-1}} \xi_{r_{1}}} \\
\vdots & \vdots & \ddots & \vdots \\
e^{2 \pi i \tau \lambda_{s_{0}} \xi_{r_{L-1}}} & e^{2 \pi i \tau \lambda_{s_{1}} \xi_{1}} & \cdots & e^{2 \pi i \tau \lambda_{s_{L-1}} \xi_{r_{L-1}}}
\end{array}\right)
$$

for some finite sequences $\left(r_{j}\right)_{j=0}^{L-1}$ and $\left(s_{j}\right)_{j=0}^{L-1}$ where $0 \leq \xi_{r_{0}}<\xi_{r_{1}}<\cdots<\xi_{r_{L-1}}$. Put

$$
P_{S}(t)=\left|\begin{array}{cccc}
t^{\lambda_{s_{0}} \xi_{r_{0}}} & t^{\lambda_{s_{1}} \xi_{r_{0}}} & \cdots & t^{\lambda_{s_{L-1}} \xi_{r_{0}}} \\
t^{\lambda_{s_{0}} \xi_{r_{1}}} & t^{\lambda_{s_{1}} \xi_{r_{1}}} & \cdots & t^{\lambda_{s_{L-1}} \xi_{r_{1}}} \\
\vdots & \vdots & \ddots & \vdots \\
t^{\lambda_{s_{0}} \xi_{r_{L-1}}} & t^{\lambda_{s_{1}} \xi_{r_{L-1}}} & \cdots & t^{\lambda_{s_{L-1}} \xi_{r_{L-1}}}
\end{array}\right|
$$


Appealing to the multi-linearity of the determinant function, we obtain

$$
P_{S}(t)=\left(\prod_{j=0}^{L-1} t^{\lambda_{s_{0}} \xi_{r_{j}}}\right) \cdot \operatorname{det}\left(t^{\left(\lambda_{s_{i}}-\lambda_{s_{0}}\right) \xi_{r_{j}}}\right)_{0 \leq i \leq L-1,0 \leq j \leq L-1}
$$

where

$$
\operatorname{det}\left(t^{\left(\lambda_{s_{i}}-\lambda_{s_{0}}\right) \xi_{r_{j}}}\right)_{0 \leq i \leq L-1,0 \leq j \leq L-1}=\left|\begin{array}{cccc}
1 & t^{\left(\lambda_{s_{1}}-\lambda_{s_{0}}\right) \xi_{r_{0}}} & \cdots & t^{\left(\lambda_{s_{L-1}}-\lambda_{s_{0}}\right) \xi_{r_{0}}} \\
1 & t^{\left(\lambda_{s_{1}}-\lambda_{s_{0}}\right) \xi_{r_{1}}} & \cdots & t^{\left(\lambda_{s_{L-1}}-\lambda_{s_{0}}\right) \xi_{r_{1}}} \\
\vdots & \vdots & \ddots & \vdots \\
1 & t^{\left(\lambda_{s_{1}}-\lambda_{s_{0}}\right) \xi_{r_{L-1}}} & \cdots & t^{\left(\lambda_{s_{L-1}}-\lambda_{s_{0}}\right) \xi_{r_{L-1}}}
\end{array}\right| .
$$

Note that $\left(t^{\left(\lambda_{s_{i}}-\lambda_{s_{0}}\right) \xi_{r_{j}}}\right)_{0 \leq i \leq L-1,0 \leq j \leq L-1}$ is a generalized Vandermonde matrix. As such, [15, Page 135],

$$
\begin{aligned}
P_{S}(t) & =\left(\prod_{i>j}\left(t^{\lambda_{s_{i}}-\lambda_{s_{0}}}-t^{\lambda_{s_{j}}-\lambda_{s_{0}}}\right)\right) \times\left(\prod_{j=0}^{L-1} t^{\lambda_{s_{0}} \xi_{r_{j}}}\right) \\
& \times s_{\left(\xi_{r_{L-1}}-L+1, \xi_{r_{L-2}}-L+2, \cdots, \xi_{r_{0}}\right)}\left(t^{\lambda_{s_{0}}-\lambda_{s_{0}}}, t^{\lambda_{s_{1}}-\lambda_{s_{0}}}, \cdots, t^{\lambda_{s_{L-1}}-\lambda_{s_{0}}}\right)
\end{aligned}
$$

where $s_{\left(\xi_{r_{L-1}}-L+1, \xi_{r_{L-2}}-L+2, \cdots, \xi_{r_{0}}\right)}$ is the Schur function with positive integer coefficients where $\left(\xi_{r_{L-1}}-L+1, \xi_{r_{L-2}}-L+2, \cdots, \xi_{r_{0}}\right)$ is a partition of the positive integer $\left(\xi_{r_{L-1}}-L+1\right)+$ $\left(\xi_{r_{L-2}}-L+2\right)+\cdots+\xi_{r_{0}}$. In other words, there exists a finite sequence $I$ of $L$-tuples of non-negative integers such that

$$
\begin{aligned}
& s_{\left(\xi_{r_{L-1}}-L+1, \xi_{r_{L-2}}-L+2, \cdots, \xi_{r_{0}}\right)}\left(t^{\lambda_{s_{0}}-\lambda_{s_{0}}}, t^{\lambda_{s_{1}}-\lambda_{s_{0}}}, \cdots, t^{\lambda_{s_{L-1}}-\lambda_{s_{0}}}\right) \\
& =\sum_{\left(m_{0}, m_{1} \cdots, m_{L-1}\right) \in I} t^{m_{0}\left(\lambda_{s_{0}}-\lambda_{s_{0}}\right)+m_{1}\left(\lambda_{s_{1}}-\lambda_{s_{0}}\right)+\cdots+m_{L-1}\left(\lambda_{s_{L-1}}-\lambda_{s_{0}}\right)}
\end{aligned}
$$

where each integer $m_{j}$ represents the number of times the $j$-th variable of the Schur function, occurs in the $\left(\xi_{r_{L-1}}-L+1, \xi_{r_{L-2}}-L+2, \cdots, \xi_{r_{0}}\right)$-tableaux. Thus,

$$
\begin{aligned}
P_{S}(t) & =\left(\prod_{j=0}^{L-1} t^{\lambda_{s_{0}} \xi_{r_{j}}}\right) \times \prod_{i>j}\left(t^{\lambda_{s_{i}}-\lambda_{s_{0}}}-t^{\lambda_{s_{j}}-\lambda_{s_{0}}}\right) \\
& \times\left(\sum_{\left(m_{0}, m_{1} \cdots, m_{L-1}\right) \in I} t^{m_{0}\left(\lambda_{s_{0}}-\lambda_{s_{0}}\right)+m_{1}\left(\lambda_{s_{1}}-\lambda_{s_{0}}\right)+\cdots+m_{L-1}\left(\lambda_{s_{L-1}}-\lambda_{s_{0}}\right)}\right)
\end{aligned}
$$

and in light of the discussion above, given a positive real number $r$, it is clear that $P_{S}(r)$ is not equal to zero since

$$
\sum_{\left(m_{0}, m_{1} \cdots, m_{L-1}\right) \in I} r^{m_{0}\left(\lambda_{s_{0}}-\lambda_{s_{0}}\right)+m_{1}\left(\lambda_{s_{1}}-\lambda_{s_{0}}\right)+\cdots+m_{L-1}\left(\lambda_{s_{L-1}}-\lambda_{s_{0}}\right)}>0
$$

and we conclude that $P_{S}$ is a nonzero polynomial in the variable $t$. Next, since

$$
|\operatorname{det}(S)|=\left|\operatorname{det}\left(\left(e^{2 \pi i \tau \lambda_{s_{k}} \xi_{r_{j}}}\right)_{0 \leq j \leq L-1,0 \leq k \leq L-1}\right)\right|=\left|P_{S}\left(e^{2 \pi i \tau}\right)\right|
$$


under the assumption that $e^{2 \pi i \tau}$ is a transcendental number, it follows that $\operatorname{det}(S)=$ $P_{S}\left(e^{2 \pi i \tau}\right) \neq 0$. Otherwise, let $Q$ be the least common multiple of all polynomials $P_{S}$ where $S$ runs through all possible square submatrices of $\mathcal{M}$. Next, let $\tau$ be a real number such that $e^{2 \pi i \tau}$ is an algebraic complex number which is not a root of the polynomial $Q$. Fixing such a real number $\tau$, we obtain that every minor of $\mathcal{M}_{\tau}$ is not equal to zero.

Example 25. Let

$$
D=\left(\begin{array}{cccc}
e^{2 \pi i \sqrt{2}} & 0 & 0 & 0 \\
0 & e^{4 \pi i \sqrt{2}} & 0 & 0 \\
0 & 0 & e^{6 \pi i \sqrt{2}} & 0 \\
0 & 0 & 0 & e^{8 \pi i \sqrt{2}}
\end{array}\right), T=\left(\begin{array}{cccc}
0 & 0 & 0 & 1 \\
1 & 0 & 0 & 0 \\
0 & 1 & 0 & 0 \\
0 & 0 & 1 & 0
\end{array}\right), f=\left(\begin{array}{l}
1 \\
2 \\
3 \\
4
\end{array}\right) .
$$

With the help of a Computer Algebra System, we were able to verify that

$$
\left\{D^{k} T^{j} f: j \in \mathbb{Z}_{4}, k \in\{0,1, \cdots, 6\}\right\}
$$

is a full spark frame with upper and lower frame bounds roughly around 258 and 174 respectively.

\section{REFERENCES}

[1] Boris Alexeev, Jameson Cahill, and Dustin G. Mixon. Full spark frames. J. Fourier Anal. Appl., 18(6):1167-1194, 2012.

[2] Marcus Appleby, Steven Flammia, Gary McConnell, and Jon Yard. SICs and algebraic number theory. Found. Phys., 47(8):1042-1059, 2017.

[3] Jameson Cahill, Dustin G. Mixon, and Nate Strawn. Connectivity and irreducibility of algebraic varieties of finite unit norm tight frames. SIAM J. Appl. Algebra Geom., 1(1):38-72, 2017.

[4] Peter G. Casazza. The Kadison-Singer and Paulsen problems in finite frame theory. In Finite frames, Appl. Numer. Harmon. Anal., pages 381-413. Birkhäuser/Springer, New York, 2013.

[5] Peter G. Casazza and Gitta Kutyniok, editors. Finite frames. Applied and Numerical Harmonic Analysis. Birkhäuser/Springer, New York, 2013. Theory and applications.

[6] Ole Christensen. An introduction to frames and Riesz bases. Applied and Numerical Harmonic Analysis. Birkhäuser/Springer, [Cham], second edition, 2016.

[7] A. Dvoretzky and Th. Motzkin. A problem of arrangements. Duke Math. J., 14:305-313, 1947.

[8] RJ Evans and IM Isaacs. Generalized vandermonde determinants and roots of unity of prime order. Proceedings of the American Mathematical Society, 58(1):51-54, 1976.

[9] Gerald B Folland. A course in abstract harmonic analysis. Chapman and Hall/CRC, 2016.

[10] Karlheinz Gröchenig. Foundations of time-frequency analysis. Applied and Numerical Harmonic Analysis. Birkhäuser Boston, Inc., Boston, MA, 2001.

[11] Jim Lawrence, Götz E. Pfander, and David Walnut. Linear independence of Gabor systems in finite dimensional vector spaces. J. Fourier Anal. Appl., 11(6):715-726, 2005.

[12] Romanos-Diogenes Malikiosis. A note on Gabor frames in finite dimensions. Applied and Computational Harmonic Analysis, 38(2):318-330, 2015.

[13] Romanos-Diogenes Malikiosis. Spark deficient Gabor frames. Pac. J. Math., 294(1):159-180, 2018.

[14] Adam W. Marcus, Daniel A. Spielman, and Nikhil Srivastava. Ramanujan graphs and the solution of the Kadison-Singer problem. In Proceedings of the International Congress of Mathematicians-Seoul 2014. Vol. III, pages 363-386. Kyung Moon Sa, Seoul, 2014.

[15] Vadim Olshevsky. Structured matrices in mathematics, computer science, and engineering: proceedings of an AMS-IMS-SIAM joint summer research conference, University of Colorado, Boulder, June 27-July 1, 1999, volume 280. American Mathematical Soc., 2001.

[16] Vignon Oussa. Dihedral group frames which are maximally robust to erasures. Linear and Multilinear Algebra, 63(12):2494-2510, 2015. 
[17] Vignon Oussa and Brian Sheehan. Dihedral group frames with the haar property. Linear and Multilinear Algebra, 66(6):1121-1132, 2018.

[18] Ambar N. Sengupta. Representing finite groups. Springer, New York, 2012. A semisimple introduction.

[19] Shayne FD Waldron. Group frames. In An Introduction to Finite Tight Frames, pages 209-243. Springer, 2018.

[20] Gerhard Zauner. Quantum designs: foundations of a noncommutative design theory. Int. J. Quantum Inf., 9(1):445-507, 2011. 